\title{
THE SECOND MULTIPLICATIVE ZAGREB ECCENTRICITY INDEX OF CIRCUMCORONENE SERIES OF BENZENOID
}

\author{
Mohammad Reza Farahani ${ }^{a}$ and Wei Gao \\ aDepartment of Applied Mathematics, Iran University of Science and Technology \\ (IUST), Narmak, Tehran 16844, Iran \\ ${ }^{\mathrm{b}}$ School of Information Science and Technology, Yunnan Normal University, \\ Kunming 650500, P. R. China
}

\begin{abstract}
Let $G=(V, E)$ be a simple connected graph. The sets of vertices and edges of $G$ are denoted by $V=V(G)$ and $E=E(G)$, respectively. The first and second Zagreb indices are equal to $M_{1}(G)=\sum_{\nu \in V(G)} d_{\nu}^{2}$ and $M_{2}(G)=\sum_{u \nu \in E(G)}\left(d_{u} \times d_{\nu}\right)$, respectively. The first and second multiplicative Zagreb eccentricity indices of $G$ are defined, respectively, as $\Pi E_{1}(G)=\prod_{\nu \in V(G)} \varepsilon(\nu)^{2}$ and $\Pi E_{2}(G)=\prod_{u \nu \in E(G)} \varepsilon(\nu) \times \varepsilon(u)$, respectively, where $\varepsilon(\nu)$ is the eccentricity of vertex $v$ in graph $G$.

In this paper, we focus on the structure of molecular graph circumcoronene series of benzenoid $H_{k}(k \geq 1)$ and present a closed formula for the second multiplicative Zagreb eccentricity index of $H_{k}$.
\end{abstract}

\footnotetext{
${ }^{*}$ Corresponding author.

E-mail address: mrfarahani88@gmail.com (Mohammad Reza Farahani).
}

Copyright (C) 2016 Scientific Advances Publishers

2010 Mathematics Subject Classification: 05C05, 05C12.

Submitted by Jianqiang Gao.

Received December 1, 2015; Revised December 14, 2015 
Keywords: Zagreb indices, second multiplicative Zagreb eccentricity index, circumcoronene series of benzenoid.

\section{Introduction}

Let $G=(V, E)$ be a simple connected graph of finite order $n=|V|=|V(G)|$ with the set of vertices and the set of edges $E=E(G)$. A general reference for the notation in graph theory is [1].

For every vertex $\nu \in V(G)$, we denote the degree of $\nu$ by $d_{\nu}$ which the degree of any vertex is the number of first neighbour of $\nu$. Let the maximum and minimum degree of all the vertices of $G$ are, respectively, denoted by $\Delta$ and $\delta$. The distance $d(u, v)$ between the vertices $u$ and $v$ of the graph $G$ is equal to the length of (number of edges in) the shortest path that connects $u$ and $\nu$. The eccentricity of a vertex is the distance between $v$ and a vertex farthest from $\nu$ and is denoted by $\varepsilon(\nu)$ thus $\varepsilon(\nu)=\operatorname{Max}\{d(u, v) \mid \forall u \in V(G)\}$.

Let the maximum and minimum eccentricity over all vertices of $G$ are called the diameter and radius of $G$ and denoted by $D(G)$ and $R(G)$, respectively.

In other words,

$$
\begin{gathered}
D(G)=\operatorname{Max}_{\nu \in V(G)}\{d(u, \nu) \mid \forall u \in V(G)\}, \\
R(G)=\operatorname{Min}_{\nu \in V(G)}\{\operatorname{Max}\{d(u, \nu) \mid \forall u \in V(G)\}\} .
\end{gathered}
$$

Mathematical chemistry is a branch of theoretical chemistry for discussion and prediction of the molecular structure using mathematical methods without necessarily referring to quantum mechanics. Chemical graph theory is a branch of mathematical chemistry which applies graph theory to mathematical modelling of chemical phenomena. A molecular graph is a simple finite graph such that its vertices correspond to the 
atoms and the edges to the bonds. In chemistry, graph invariants are known as topological indices. In graph theory, we have many different topological indices of arbitrary graph $G$.

A topological index is a real number related to a molecular graph, it does not rely on the labelling or the pictorial representation of a graph which is invariant under graph automorphisms. Obviously, every topological index defines a counting polynomial and vice versa [1-3].

One of the best known and widely used is the Zagreb topological index and was defined about forty years ago by Gutman and Trinajsti in 1972 [4] (or, more precisely, the first Zagreb index, because there exists also a second Zagreb index [3]). The first Zagreb index of $G$ is defined as the sum of the squares of the degrees of all vertices of $G$ [5]. The first and second Zagreb indices of $G$ are denoted by $M_{1}(G)$ and $M_{2}(G)$, respectively, and defined as follows:

$$
\begin{aligned}
& M_{1}(G)=\sum_{\nu \in V(G)} d_{\nu}^{2}, \\
& M_{2}(G)=\sum_{u \nu \in E(G)}\left(d_{u} \times d_{\nu}\right),
\end{aligned}
$$

where $d_{u}$ and $d_{\nu}$ are the degrees of $u$ and $\nu$, respectively. The first and second Zagreb indices $M_{1}$ and $M_{2}$ are applied to study molecular, chirality in quantitative structure-activity relationship (QSAR) and quantitative structure-property relationship (QSPR) analysis, etc.

In 2012, the multiplicative variant of Zagreb indices was introduced by Todeschini et al. [6, 7], Gutman in [8] call these indices as multiplicative Zagreb indices. They are defined as

$$
\begin{aligned}
& \prod M_{1}(G)=\prod_{u \nu \in E(G)} d_{\nu}^{2}, \\
& \prod M_{2}(G)=\prod_{u \nu \in E(G)}\left(d_{u} \times d_{\nu}\right) .
\end{aligned}
$$


The eccentric version of the first Zagreb index was introduced by Ghorbani et al. [9] and Vukicevic et al. [10] in 2012 as follows:

$$
\begin{aligned}
& M_{1}^{*}(G)=\sum_{\nu \in V(G)} \varepsilon(\nu)^{2}, \\
& M_{2}^{*}(G)=\sum_{u \nu \in E(G)} \varepsilon(\nu) \times \varepsilon(u),
\end{aligned}
$$

where $\varepsilon(\nu)$ and $\varepsilon(u)$ is the eccentricity of vertices $v$ and $u$, respectively [11-13].

Recently in 2012, the multiplicative Zagreb eccentricity indices of the connected graph $G$ were introduced by Nilanjan De [14] and are defined, respectively, as follows:

$$
\begin{gathered}
\prod E_{1}(G)=\prod_{u \nu \in E(G)} \varepsilon(\nu)^{2}, \\
\prod E_{2}(G)=\prod_{u \nu \in E(G)} \varepsilon(\nu) \times \varepsilon(u) .
\end{gathered}
$$

For further results of the Zagreb eccentricity indices, we encourage the reader refer to [15-24].

In this paper, we focus on the structure of famous benzenoid molecular graph "circumcoronene series of benzenoid $H_{k}(k \geq 1)$ " and present a closed formula for the second multiplicative Zagreb eccentricity index of $H_{k}$.

\section{Main Results and Discussion}

The goal of this section is to study the multiplicative Zagreb eccentricity indices of circumcoronene series of benzenoid $H_{k}(k \geq 1)$. The circumcoronene series of benzenoid is family of benzenoid molecular graph, which consist several copy of $C_{6}$ on circumference and are generalizations of benzene molecule and the benzene molecule is a usual molecule in chemistry, physics and nano sciences and is very useful to 
synthesize aromatic compounds. The general representations and first terms of this benzenoid series are $H_{1}=$ benzene, $H_{2}=$ coronene, $H_{3}=$ circumcoronene, $H_{4}=$ circumcircumcoronene are shown Figures 1,2 , and 3. This benzenoid family is presented in many papers; please refer to [24-36].
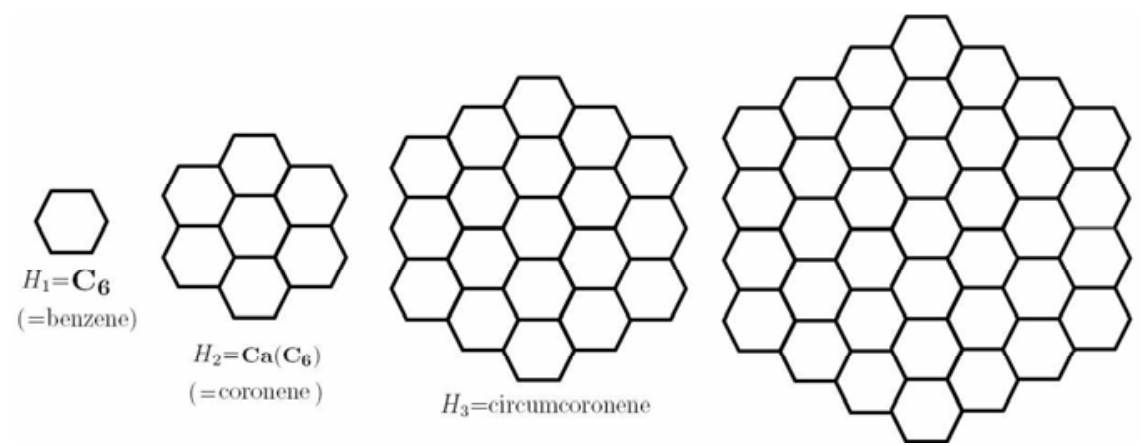

Figure 1. $H_{1}=$ benzene, $H_{2}=$ coronene, $H_{3}=$ circumcoronene, $H_{4}=$ circum circumcoronene [33-36]. 


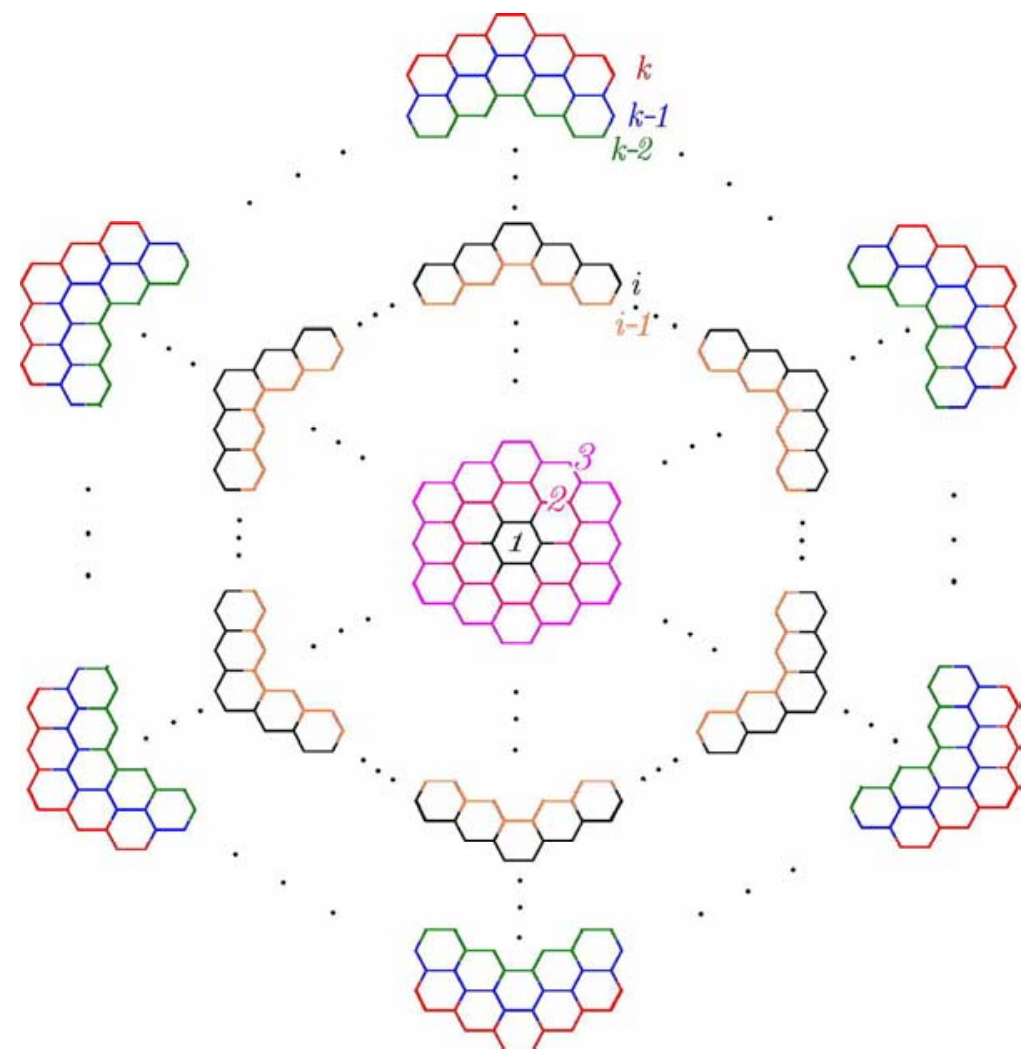

Figure 2. The ring-cuts of circumcoronene series of benzenoid $H_{k}(k \geq 1)$ [33-36].

Theorem 1. Let $H_{k}$ be the benzenoid molecular graph "circumcoronene series of benzenoid $H_{k}{ }^{\prime} \forall k \geq 1$. Then the second multiplicative Zagreb eccentricity index of $H_{k}$

$$
\Pi E_{2}\left(H_{k}\right)=2^{6(3 i-2)}(2 k+2 i-1)^{6(3 i+2 k-2)}\left(\prod_{i=1}^{k}\left[(k+i)^{6 i}(k+i-1)^{12(i-1)}\right]\right) .
$$


Proof. Consider the circumcoronene series of benzenoid $H_{k}(k \geq 1)$. Let $V\left(H_{k}\right)=\left\{\gamma^{i} z, j, \beta^{i} z, j \mid i \in \mathbb{Z}_{k} \& j \in \mathbb{Z}_{i} \& z \in \mathbb{Z}_{6}\right\}$ be a notation for vertices/atoms of $H_{k}$ and alternatively $E\left(H_{k}\right)=\left\{\gamma_{z, j}^{i} \beta_{z, j}^{i}, \gamma^{i} z, j+1 \beta^{i} z, j\right.$, $\gamma^{i-1} z, j \beta^{i} z, j$ and $\left.\gamma_{z, j}^{i} \gamma^{i} z, j+1 / i \in \mathbb{Z}_{k} \& j \in \mathbb{Z}_{i} \& z \in \mathbb{Z}_{6}\right\}$ be a notation for edges/bonds of $H_{k}$.

So, from the general structure of $H_{k}$ in Figures 2 and 3, it is easy to see that the circumcoronene series of benzenoid has $6 k^{2}$ vertices/atoms and there are $9 k^{2}-3 k$ edges/bonds in general case of this benzenoid graph. Since

$$
\begin{aligned}
\left|V\left(H_{k}\right)\right| & =\sum_{i=1}^{k} \sum_{j=1}^{i} \sum_{z=1}^{6} \gamma_{z, j}^{i}+\sum_{i=2}^{k} \sum_{j=1}^{i} \sum_{z=1}^{6} \beta_{z, j}^{i}=6 \sum_{i=1}^{k} i+6 \sum_{i=0}^{k-1} i=6 k^{2} \\
\left|E\left(H_{k}\right)\right| & =\sum_{i=1}^{k} \sum_{j=1}^{i} \sum_{z=1}^{6}\left(\gamma^{i} z, j \beta_{z, j}^{i}+\gamma_{z, j+1}^{i} \beta_{z, j}^{i}+\gamma^{i-1} z, j \beta_{z, j}^{i}+\gamma_{z, j}^{i} \gamma_{z, j+1}^{i}\right) \\
& =6 \sum_{i=1}^{k-1} i+6 \sum_{i=1}^{k-1} i+6 \sum_{i=1}^{k-1} i+6 k=9 k^{2}-3 k
\end{aligned}
$$

Now, to achieve our aims, we should use the ring-cut method. The ring-cut method is a modify version of the cut method that divide all vertices and edges of a graph $G$ into several partitions (or, more precisely, cuts) with similar mathematical and topological properties. For further study and results of the cut method and the ring-cut method, we encourage the reader refer to [27, 28, 36].

Also, for example, reader can see the vertex/edge notations and ringcuts of circumcoronene series of benzenoid in Figures 2 and 3.

Now, by according to Figures 2 and 3 and using the results from [36], we can calculate the eccentric $\varepsilon$ of all vertices of circumcoronene series of benzenoid $H_{k}, \forall k \geq 1$, such that $\forall i=1, \ldots, k$ and $\forall j \in \mathbb{Z}_{i} \& \forall z \in \mathbb{Z}_{6}$ as follows: 


$$
\begin{aligned}
\forall i=1, \ldots, k ; j \in \mathbb{Z}_{i-1} \& z & \in \mathbb{Z}_{6}: \varepsilon\left(\beta_{z, j}^{i}\right)=\underbrace{d\left(\beta_{z, j}^{i}, \beta_{z+3, j}^{i}\right)}_{4 i-3} \\
& +\underbrace{d\left(\beta_{z+3, j}^{i}, \gamma_{z+3, j}^{k}\right)}_{2(k-i)+1}=2(k+1)-2,
\end{aligned}
$$

$\forall i=1, \ldots, k ; j \in \mathbb{Z}_{i} \& z \in \mathbb{Z}_{6}: \varepsilon\left(\gamma^{i} z, j\right)=\underbrace{d\left(\gamma_{z, j}^{i}, \gamma_{z+3, j}^{i}\right)}_{4 i-1}$

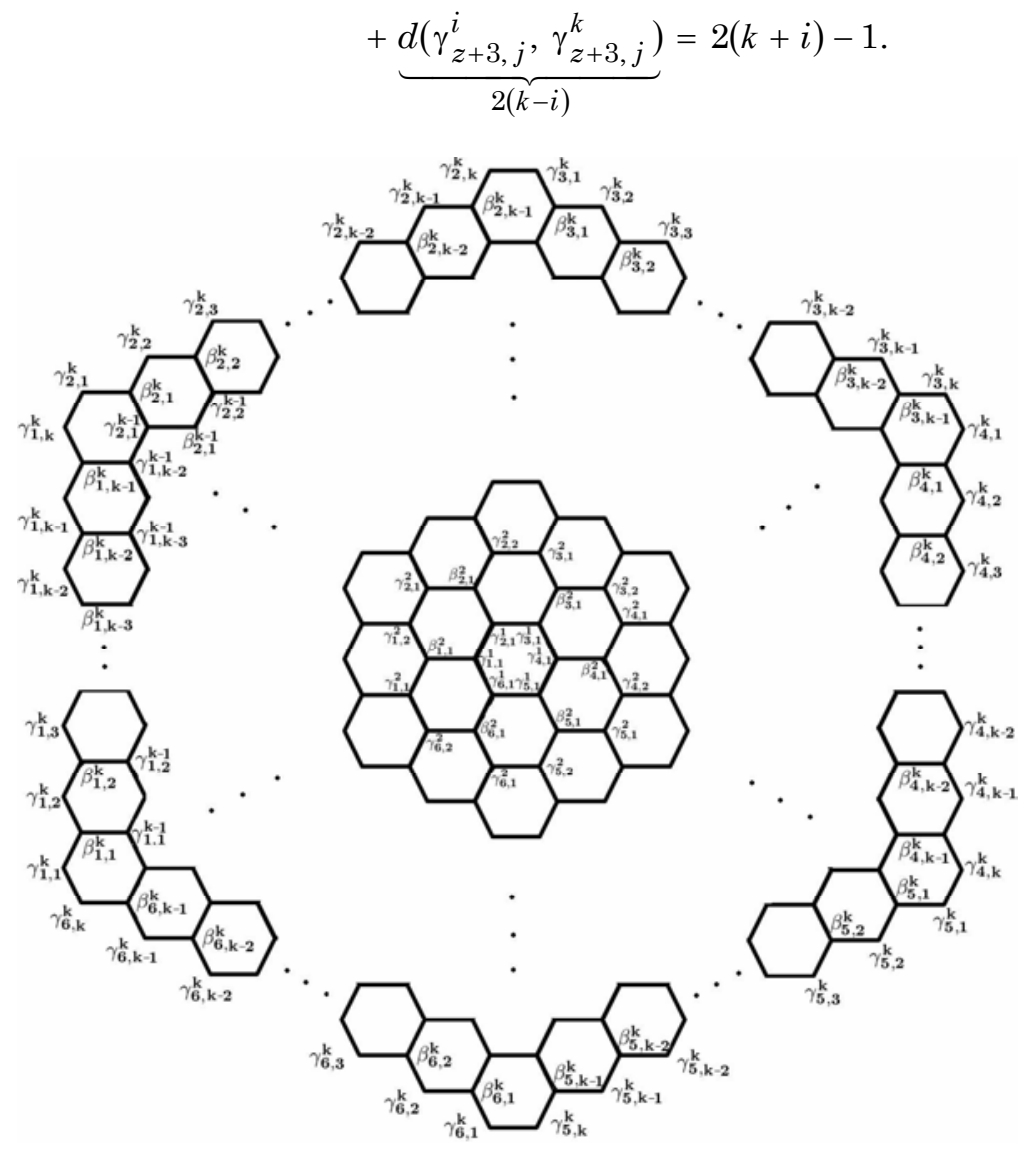

Figure 3. The general representation of circumcoronene series of benzenoid $H_{k}(\forall k \geq 1)$ [33-36]. 
Thus by according to Figures 2 and 3 and above results, we have following computations for the second multiplicative Zagreb eccentricity index of circumcoronene series of benzenoid $H_{k}(k \geq 1)$ as

$$
\begin{aligned}
\Pi E_{2}\left(H_{k}\right)= & \prod_{u \nu \in E\left(H_{k}\right)}(\varepsilon(\nu) \times \varepsilon(u)) \\
= & \left(\prod_{\beta_{z, j}^{i} \gamma_{z, j}^{i} \in E\left(H_{k}\right)} \varepsilon\left(\beta_{z, j}^{i}\right) \varepsilon\left(\gamma_{z, j}^{i}\right)\right) \times\left(\prod_{\beta_{z, j}^{i} \gamma_{z, j+1}^{i} \in E\left(H_{k}\right)} \varepsilon\left(\beta_{z, j}^{i}\right) \varepsilon\left(\gamma_{z, j+1}^{i}\right)\right) \\
& \times\left(\prod_{\beta_{z, j}^{i} \gamma_{z, j}^{i-1} \in E\left(H_{k}\right)} \varepsilon\left(\beta_{z, j}^{i+1}\right) \varepsilon\left(\gamma_{z, j}^{i-1}\right)\right) \times\left(\prod_{\gamma_{z, j}^{i}}^{i} \prod_{z+1,1} \in E\left(H_{k}\right)\right. \\
= & \prod_{z=1}^{6}\left(\prod_{i=2}^{k} \varepsilon\left(\gamma_{z, i}^{i}\right) \varepsilon\left(\gamma_{z+1,1}^{i}\right)\right) \\
& \left.\times \prod_{z=1}^{6} \varepsilon\left(\prod_{i=1}^{i}\left(\prod_{z, j}^{k-1}\right) \varepsilon\left(\gamma_{z, j}^{i}\right)\right) \times \prod_{z=1}^{6} \varepsilon\left(\prod_{i=2}^{k} \varepsilon\left(\prod_{z, j}^{i+1}\right) \varepsilon\left(\gamma_{z, j}^{i-1}\right)\right) \times \prod_{j=1}^{i} \varepsilon\left(\prod_{z, j}^{i}\right) \varepsilon\left(\gamma_{z, j+1}^{i}\right)\right)
\end{aligned}
$$

Now, we can insert the equivalences of eccentric $\varepsilon\left(\beta^{i} z, j\right)$ and $\varepsilon\left(\gamma^{i} z, j\right)$ $\left(\forall i=1, \ldots, k ; j \in \mathbb{Z}_{i-1} \& z \in \mathbb{Z}_{6}\right)$ by their exact value $2(k+i)-2$ and $2(k+i)-1$, respectively.

$$
\begin{aligned}
\Pi E_{2}\left(H_{k}\right)= & \prod_{i=2}^{k}[(2 k+2 i-1)(2 k+2 i-2)]^{6(i-1)} \\
& \times \prod_{i=2}^{k}[(2 k+2 i-1)(2 k+2 i-2)]^{6(i-1)} \\
& \times \prod_{i=1}^{k}[(2 k+2 i-1)(2 k+2 i)]^{6 i} \times \prod_{i=1}^{k}\left[(2 k+2 i-1)^{2}\right]^{6}
\end{aligned}
$$




$$
\begin{aligned}
= & \left(\frac{\prod_{i=1}^{k}[(2 k+2 i-1)(2 k+2 i-2)]^{12(i-1)}}{(2 k+1)(2 k)^{0}=1}\right) \\
& \times \prod_{i=1}^{k}[2(2 k+2 i-1)(k+i)]^{6 i} \times \prod_{i=1}^{k}[(2 k+2 i-1)]^{12} \\
= & 2(2 k+2 i-1)^{12(i-1)} \times 2(2 k+2 i-1)^{6 i} \times(2 k+2 i-1)^{12 k} \\
& \times\left(\prod_{i=1}^{k}[(k+i-1)]^{12(i-1)} \times \prod_{i=1}^{k}[(k+i)]^{6 i}\right) \\
= & 2^{6(3 i-2)}(2 k+2 i-1)^{6(3 i+2 k-2)}\left(\prod_{i=1}^{k}\left[(k+i)^{6 i}(k+i-1)^{12(i-1)}\right]\right) .
\end{aligned}
$$

Finally, the second multiplicative Zagreb eccentricity index of $H_{k}, \forall k \in \mathbb{Z}$ is equal to

$$
\Pi E_{2}\left(H_{k}\right)=2^{6(3 i-2)}(2 k+2 i-1)^{6(3 i+2 k-2)}\left(\prod_{i=1}^{k}\left[(k+i)^{6 i}(k+i-1)^{12(i-1)}\right]\right) .
$$

Here, we complete the proof of the Theorem 1.

\section{References}

[1] D. B. West, An Introduction to Graph Theory, Prentice-Hall, 1996.

[2] A. T. Balaban (Ed.), From Chemical Topology to Three-dimensional Geometry, Plenum, New York, 1997.

[3] R. Todeschini and V. Consonni, Handbook of Molecular Descriptors, Wiley, Weinheim, 2000. 
[4] I. Gutman and N. Trinajstić, Graph theory and molecular orbitals, III, Total $\pi$-electron energy of alternant hydrocarbons, Chem. Phys. Lett. 17 (1972), 535-538.

[5] I. Gutman and K. C. Das, The first Zagreb index 30 years after, MATCH Commun. Math. Comput. Chem. 50 (2004), 83-92.

[6] R. Todeschini and V. Consonni, New local vertex invariants and molecular descriptors based on functions of the vertex degrees, MATCH Commun. Math. Comput. Chem. 64 (2010), 359-372.

[7] R. Todeschini, D. Ballabio and V. Consonni, Novel molecular descriptors based on functions of the vertex degrees, in: I. Gutman and B. Furtula (Eds.), Novel Molecular Structure Descriptors-Theory and Applications I, Univ. Kragujevac, Kragujevac, (2010), 73-100.

[8] I. Gutman, Multipicative Zagreb indices of trees, Bull. Internat. Math. Virt. Inst. 1 (2011), 13-19.

[9] M. Ghorbani and M. A. Hosseinzadeh, A new version of Zagreb indices, Filomat 26(1) (2012), 93-100.

[10] D. Vukicevic and A. Graovac, Note on the comparison of the first and second normalized Zagreb eccentricity indices, Acta Chim. Slov. 57 (2010), 524-528.

[11] S. Sardana and A. K. Madan, MATCH Commun. Math. Comput. Chem. 43(85) (2001).

[12] S. Gupta, M. Singh and A. K. Madan, J. Math. Anal. Appl. (2002), 266-259.

[13] B. Furtula, A. Graovac and D. Vukičević, Disc. Appl. Math. 157 (2009), 2828.

[14] N. De, On multiplicative Zagreb eccentricity indices, South Asian J. Math. 2(6) (2012), 570-577.

[15] Z. Du, B. Zhou and N. Trinajstic, Extremal properties of the Zagreb eccentricity indices, Croat. Chem. Acta 85(3) (2012), 359-362.

[16] K. C. Das, D. W. Lee and A. Graovac, Some properties of the Zagreb eccentricity indices, Ars Math. Contemp. 6 (2013), 117-125.

[17] K. Xu and H. Hua, A united approach to extremal multiplicative Zagreb indices for trees, unicyclic and bicyclic graphs, MATCH Commun. Math. Comput. Chem. 68 (2012), 241-256.

[18] J. Liu and Qianhong Zhang, Sharp upper bounds for multiplicative Zagreb indices, MATCH Commun. Math. Comput. Chem. 68 (2012), 231-240.

[19] T. Reti and I. Gutman, Relations between ordinary and multiplicative Zagreb indices, Bull. Internat. Math. Virt. Inst. 2 (2012), 133-140.

[20] Z. Luo and J. Wu, Multiplicative Zagreb eccentricity indices of some composite graphs, Trans. Comb. 3(2) (2014), 21-29.

[21] H. Wang and H. Bao, A note on multiplicative sum Zagreb index, South Asian J. Math. 2(6) (2012), 578-583. 
[22] M. R. Farahani, Multiplicative versions of Zagreb indices of $T U S C_{4} C_{8}(S)$, Journal of Chemistry and Materials Research 2(2) (2015), 67-70.

[23] M. R. Farahani, On multiple Zagreb indices of dendrimer nanostars, International Letters of Chemistry, Physics and Astronomy 52 (2015), 147-151.

[24] M. R. Farahani, On multiple Zagreb indices of circumcoronene homologous series of benzenoid, Chemical Physics Research Journal 7(2) (2014), 277-282.

[25] V. Chepoi and S. Klavžar, Distances in benzenoid systems: Further developments, Discrete. Math. 192 (1998), 27-39.

[26] S. Klavžar and I. Gutman, Bounds for the Schultz molecular topological index of benzenoid systems in terms of Wiener index, J. Chem. Inf. Comput. Sci. 37(4) (1997), 741-744.

[27] S. Klavžar, A bird s eye view of the cut method and a survey of its applications in chemical graph theory, MATCH Commun. Math. Comput. Chem. 60 (2008), 255-274.

[28] P. E. John, P. V. Khadikar and J. Singh, A method of computing the PI index of benzenoid hydrocarbons using orthogonal cuts, J. Math. Chem. 42(1) (2007), 27-45.

[29] S. Klavžar, I. Gutman and B. Mohar, Labeling of benzenoid systems which reflects the vertex-distance relations, J. Chem. Inf. Comput. Sci. 35 (1995), 590-593.

[30] S. Klavžar and I. Gutman, A comparison of the Schultz molecular topological index with the Wiener index, J. Chem. Inf. Comput. Sci. 36 (1996), 1001-1003.

[31] K. Salem, S. Klavžar and I. Gutman, On the role of hyper-cubes in the resonance graphs of benzenoid graphs, Discrete Math. 13(8) (2003), 306.

[32] P. Zigert, S. Klavžar and I. Gutman, Calculating the hyper-Wiener index of benzenoid hydrocarbons, ACH Models Chem. 137 (2000), 83-94.

[33] M. R. Farahani, Computing first-, second -connectivity index and first-, second-sumconnectivity index of circumcoronene series of benzenoid, Pacific Journal of Applied Mathematics 6(4) (2014).

[34] M. R. Farahani, A new version of Zagreb index of circumcoronene series of benzenoid, Chemical Physics Research Journal 6(1) (2013), 27-33.

[35] M. R. Farahani, Third-connectivity and third-sum-connectivity indices of circumcoronene series of benzenoid $H_{k}$, Acta Chim. Slov. 60 (2013), 198-202.

[36] M. R. Farahani, Computing eccentricity connectivity polynomial of circumcoronene series of benzenoid $H_{k}$ by ring-cut method, Annals of West University of TimisoaraMathematics and Computer Science 51(2) (2013), 29-37. 